\title{
Heart rate variability and biomarkers of systemic inflammation in patients with stable coronary heart disease: findings from the Heart and Soul Study
}

\author{
Roland von Känel • Robert M. Carney • \\ Shoujun Zhao Mary A. Whooley
}

Received: 17 April 2010/Accepted: 7 September 2010/Published online: 21 September 2010

(C) Springer-Verlag 2010

\begin{abstract}
Background Chronic low-grade systemic inflammation is a key component in atherogenesis. Decreased heart rate variability (HRV), a strong predictor of cardiovascular events, has been associated with elevations in circulating levels of C-reactive protein (CRP), interleukin (IL)-6, and fibrinogen in apparently healthy individuals. We investigated whether decreased HRV is associated with inflammatory markers in patients with coronary heart disease (CHD).

Methods We studied the relationship between HRV and CRP, IL-6, and fibrinogen in 862 outpatients with CHD. All participants provided fasting blood samples and underwent 24-h ambulatory monitoring to assess timedomain measures of HRV (MeanNN, SDNN, SDANN, and RMSSD). Regression analyses were adjusted for age, sex,
\end{abstract}

R. von Känel $(\bowtie)$

Department of General Internal Medicine, Division of Psychosomatic Medicine, Inselspital, Bern University

Hospital, University of Bern, 3010 Bern, Switzerland

e-mail: roland.vonkaenel@insel.ch

\section{R. M. Carney}

Department of Psychiatry, Washington University

School of Medicine, St. Louis, MO, USA

S. Zhao

Department of Epidemiology and Biostatistics,

University of California, San Francisco, USA

\section{A. Whooley}

Section of General Internal Medicine,

San Francisco VA Medical Center, San Francisco, USA

M. A. Whooley

Department of Medicine, University of California,

San Francisco, USA ethnicity, body mass index, smoking, diabetes, beta blocker use, and cardiopulmonary history.

Results MeanNN, SDNN, and SDANN were significantly and inversely associated with CRP and IL-6 levels in ageadjusted models and after adjustment for all covariates $(p \leq 0.02)$. MeanNN, SDNN, and SDANN were also inversely associated with fibrinogen levels in age-adjusted models $(p<0.03)$, but not significantly so in multivariable-adjusted models. Reduced vagal modulation of heart rate (RMSSD) was not significantly associated with any inflammatory measures.

Conclusions Reduced cardiac autonomic control is associated with increased systemic inflammation in patients with stable CHD. This relationship was largely independent of important covariates.

Keywords Autonomic nervous system - Biomarkers · Cardiac autonomic function - Cardiovascular disease . Heart rate variability $\cdot$ Inflammation

\section{Introduction}

Chronic low-grade systemic inflammation is involved in all stages of coronary atherogenesis, from initial endothelial dysfunction to plaque rupture and clinical manifestation of acute atherothrombotic events [1]. Meta-analyses show that elevated levels of biomarkers of systemic inflammation, particularly C-reactive protein (CRP), interleukin (IL)-6, and fibrinogen, predict incident coronary heart disease (CHD), independent of traditional cardiovascular risk factors [2-4]. Inflammatory biomarkers are also predictive of cardiovascular outcomes in patients with established CHD [5]. For instance, in patients with stable CHD, increased CRP levels predict unstable angina, recurrent non-fatal 
myocardial infarction (MI), and fatal coronary events [68]. Elevated fibrinogen levels predict non-fatal MI and cardiovascular death in patients with chronic CHD [9], and elevated IL-6 during unstable angina increases the risk of in-hospital coronary events [10].

Additional research suggests a relationship between compromised autonomic regulation of the heart, as assessed by decreased heart rate variability (HRV), and increased systemic inflammation [11]. In apparently healthy individuals of varying age, several indices of decreased HRV have been associated with elevated circulating levels of CRP [12-19], IL-6 [18-20], and fibrinogen [15, 21], independent of covariates. In patients with stable CHD, however, the relationship between HRV and levels of CRP, IL-6, and fibrinogen has not been well studied. Previous studies have been small [22-26], mostly restricted to women [26, 27], and generally limited to one measure of inflammation [28]. Therefore, we sought to evaluate the relationship between cardiac autonomic control and three measures of systemic inflammation in a large sample of women and men with stable CHD. Our specific hypothesis was that decreased HRV would be associated with elevated circulating levels of CRP, IL-6, and fibrinogen.

\section{Materials and methods}

Study participants and recruitment

The Heart and Soul Study is a prospective cohort study of health outcomes in outpatients with stable CHD. The details of eligibility and enrollment have previously been prescribed [29]. In brief, between $9 / 2000$ and 12/2002, a total of 1,024 participants were recruited using administrative databases from two Department of Veterans Affairs Medical Centers (San Francisco, California and Palo Alto, CA, USA), one university medical center (University of California, San Francisco, USA), and nine public health clinics in the Community Health Network of San Francisco.

Patients were eligible to participate if they had at least one of the following: a history of MI or coronary revascularization, angiographic evidence of $\geq 50 \%$ stenosis in one or more major coronary vessels, or evidence of exercise-induced ischemia by treadmill or nuclear testing. Patients were not eligible if they had an acute coronary syndrome within the past 6 months, could not walk one block, or were planning to move from the area within 3 years.

All participants completed a daylong study appointment at the San Francisco Veterans Affairs Medical Center in California that included a comprehensive health interview, a medical history questionnaire, and 24-h ambulatory
Holter monitoring to determine HRV. Particularly, for the purpose of the present study, the fasting blood samples for the determination of biomarkers were drawn first thing in the morning (around 9 am), a few hours before the Holter monitoring was started on the same day (around $3 \mathrm{pm}$ ). We excluded 151 patients because they had a paced rhythm, atrial fibrillation, or their Holter ECG was unable to be collected, and 11 patients for whom we did not have fasting blood samples, leaving 862 participants for this cross-sectional analysis. All participants provided written informed consent to this protocol which was approved by the institutional review board at each of the sites.

\section{Additional participant characteristics}

Sociodemographic factors, medical history, smoking status, and alcohol use [30] were determined by questionnaire. We measured weight and height to calculate the body mass index (BMI, $\mathrm{kg} / \mathrm{m}^{2}$ ) Participants were considered to be physically active if they reported being somewhat, very, or extremely active (vs. not at all or a little active) in the last month. Participants were instructed to bring their medication bottles to the study appointment, and study personnel recorded current medications. We measured resting blood pressure by means of standard sphygmomanometry and assayed serum lipids after a 12-h fast.

\section{Heart rate variability}

Heart rate variability parameters were obtained via 3channel 24-h ambulatory Holter electrocardiographic recording as recommended by the Task Force of the European Society of Cardiology and the North American Society of Pacing and Electrophysiology [31]. Tapes were scanned at 500 times real time, and electrocardiography data were digitized at a sampling frequency of $128 \mathrm{~Hz}$. Computer software (General Electric Medical System Software for Holter Analysis, GE Healthcare, Waukesha, WI, USA) was used to detect and label each QRS complex using beats with normal morphologic characteristics and a cycle length less than $20 \%$ duration of the preceding cycle length. An independent and blinded reviewer processed all Holter ECGs and modified any inappropriate computer labels, with particular focus on periods with the highest and lowest average RR intervals. The annotated QRS data were processed to allow time-domain characterization, including the mean of all normal-to-normal intervals (MeanNN in $\mathrm{ms}$ ), the standard deviation of all $\mathrm{NN}$ intervals (SDNN in $\mathrm{ms}$ ), the standard deviation of the mean of NN intervals in all 5-min segments of a 24-h recording (SDANN in ms), and the square root of the mean squared differences of successive NN intervals (RMSSD in ms). 
Inflammatory biomarkers

Participants were instructed to fast for $12 \mathrm{~h}$, not to smoke for $5 \mathrm{~h}$ before the investigation, and not to take aspirin for 1 week. Patients were allowed to take all other medications as prescribed. Serum and plasma aliquots were stored at $70^{\circ} \mathrm{C}$ until analyzed.

\section{Interleukin-6}

We used the Quantikine HS IL-6 Immunoassay (R\&D Systems, Minneapolis, MN, USA) to determine the concentration of serum IL-6 with a reportable range of $0.43-8.9 \mathrm{pg} / \mathrm{mL}$. The inter-assay $\mathrm{CV}$ is $6.5-9.6 \%$, and the intra-assay $\mathrm{CV}$ is $6.9-7.8 \%$.

\section{Fibrinogen}

Plasma fibrinogen concentrations were determined by the Clauss method. Dilutions of plasma standard of known fibrinogen concentrations are clotted with a high concentration of thrombin. The participant's clotting time is proportional to his or her plasma fibrinogen concentration. The standard assay range is $60-10,000 \mathrm{mg} / \mathrm{dL}$, and the interand intra-assay $\mathrm{CVs}$ are both $<3 \%$.

\section{C-reactive protein}

Serum high-sensitivity CRP (hs-CRP) was determined using the Roche Integra assay or (owing to a change in the laboratory) the Beckman Extended Range assay in the remaining. Results from these two assays were highly correlated ( $r=0.99$ in 185 participants). The Roche Integra hs-CRP assay has an inter-assay coefficient of variation (CV) of $3.2 \%$. Lowest limit of detection is $0.25 \mathrm{mg} / \mathrm{L}$. The Beckman Extended Range hs-CRP assay has an inter-assay $\mathrm{CV}$ of $\leq 6.7 \%$, an intra-assay $\mathrm{CV}$ of $<6.2 \%$, and a reportable range of $0.20-1,140 \mathrm{mg} / \mathrm{L}$.

\section{Statistical analysis}

Level of significance was set at $p<0.05$ (two-tailed). For descriptive purposes, differences in characteristics between participants with SDNN $<100 \mathrm{~ms}$ (low HRV) and those with $\mathrm{SDNN} \geq 100 \mathrm{~ms}$ (normal HRV) were compared using Student's $t$ test for continuous variables and $\chi^{2}$ test for dichotomous variables. This cut-off for SDNN, a measure of total HRV, was previously predictive for mortality in patients after MI [31, 32]. Values of CRP, IL6 , and fibrinogen were $\log$ transformed to approximate normal distributions.

Multivariate linear regression analysis was applied to test for a relationship between HRV measures and inflammatory factors. Two models were computed. Model 1 adjusted for age, and Model 2 additionally adjusted for sex, ethnicity (White vs. non-White), current smoking (yes vs. no), BMI, use of beta blockers, history of diabetes, history of chronic heart failure $(\mathrm{CHF})$, and history of chronic obstructive pulmonary disease (COPD). We selected these covariates because they were previously shown to be associated with HRV in different studies [31] and also in an earlier analysis of subjects from the Heart and Soul Study [33].

\section{Results}

Patient characteristics

Table 1 shows the comparison in characteristics between the 264 patients with low HRV and the 598 patients with normal HRV as per a cutoff of $100 \mathrm{~ms}$ for SDNN [31]. Patients with low HRV were more likely to be female and of non-White ethnicity. Relative to patients with normal HRV, those with low HRV were more likely to have CHF, COPD, and diabetes, and to have higher BMI. There were a significantly higher number of smokers in the group with low HRV.

Relationship between heart rate variability and inflammatory measures

Table 2 shows the partial correlation coefficients with significance level for the relationship between the various HRV measures and inflammatory factors in Model 1 that adjusted for age and in the fully adjusted Model 2 with covariates age, sex, ethnicity, smoking, BMI, use of beta blocker, history of CHF, COPD, and diabetes.

\section{Model adjusting for age}

In Model 1, nearly all of the HRV measures (i.e., Mean NN, SDNN, and SDANN) showed a significant inverse relationship with CRP, IL-6, and fibrinogen levels (Table 2). Exceptions were seen for RMSSD which was not significantly associated with any of the three inflammatory measures. For illustrative purposes, Fig. 1 depicts the age-adjusted levels of IL-6, fibrinogen, and CRP in patients with low (SDNN < 100) and in those with normal HRV (all $p<0.05$ ).

\section{Fully adjusted model}

After adjusting for covariates in Model 2, the significance of results from Model 1 was maintained for CRP and IL-6 levels. However, the previously significant relationships 
Table 1 Characteristics of participants with low (SDNN $<100 \mathrm{~ms}$ ) versus normal (SDNN $\geq 100 \mathrm{~ms}$ ) heart rate variability

\begin{tabular}{|c|c|c|c|}
\hline Variable & $\begin{array}{l}\text { Low HRV } \\
(n=264)\end{array}$ & $\begin{array}{l}\text { Normal } \\
\text { HRV } \\
(n=598)\end{array}$ & $p$ value \\
\hline \multicolumn{4}{|l|}{ Demographic factors } \\
\hline Age (years) & $66 \pm 10$ & $69 \pm 11$ & 0.235 \\
\hline Male sex & $204(77)$ & $500(84)$ & 0.027 \\
\hline High school graduate & $226(86)$ & $524(88)$ & 0.416 \\
\hline Living alone & $88(33)$ & $219(37)$ & 0.353 \\
\hline \multicolumn{4}{|l|}{ Ethnicity } \\
\hline White & $137(52)$ & $363(61)$ & 0.025 \\
\hline Black & $60(23)$ & $88(15)$ & \\
\hline Asian & $32(12)$ & $73(12)$ & \\
\hline Other & $34(13)$ & $74(12)$ & \\
\hline \multicolumn{4}{|l|}{ Cardiac history } \\
\hline Prior myocardial infarction & $143(55)$ & $323(54)$ & 0.937 \\
\hline Coronary artery bypass surgery & $106(40)$ & $207(35)$ & 0.112 \\
\hline Percutaneous revascularization & $95(36)$ & $241(40)$ & 0.232 \\
\hline \multicolumn{4}{|l|}{ Comorbid conditions } \\
\hline Hypertension & $199(75)$ & $416(70)$ & 0.095 \\
\hline Stroke & $42(16)$ & $83(14)$ & 0.447 \\
\hline Chronic heart failure & $57(22)$ & $80(13)$ & 0.003 \\
\hline $\begin{array}{l}\text { Chronic obstructive } \\
\text { pulmonary disease }\end{array}$ & $57(22)$ & $79(13)$ & 0.002 \\
\hline \multicolumn{4}{|l|}{ Metabolic factors } \\
\hline History of diabetes & $102(39)$ & $119(20)$ & $<0.001$ \\
\hline Systolic blood pressure $(\mathrm{mmHg})$ & $135 \pm 22$ & $133 \pm 21$ & 0.189 \\
\hline $\begin{array}{l}\text { Low-density lipoprotein } \\
\text { cholesterol }(\mathrm{mg} / \mathrm{dL})\end{array}$ & $105 \pm 35$ & $104 \pm 32$ & 0.538 \\
\hline $\begin{array}{l}\text { High-density lipoprotein } \\
\text { cholesterol (mg/dL) }\end{array}$ & $46 \pm 15$ & $46 \pm 14$ & 0.937 \\
\hline Body mass index $\left(\mathrm{kg} / \mathrm{m}^{2}\right)$ & $28.9 \pm 5.8$ & $28.1 \pm 4.8$ & 0.026 \\
\hline \multicolumn{4}{|l|}{ Health habits } \\
\hline Current smoking & $64(24)$ & $103(17)$ & 0.017 \\
\hline Alcohol score & $2.4 \pm 2.5$ & $2.2 \pm 2.4$ & 0.147 \\
\hline Physically active & $156(59)$ & $392(66)$ & 0.075 \\
\hline \multicolumn{4}{|l|}{ Medications } \\
\hline Beta blocker & $160(61)$ & $353(59)$ & 0.664 \\
\hline Statin & $167(63)$ & $393(66)$ & 0.485 \\
\hline $\begin{array}{l}\text { Angiotensin-converting } \\
\text { enzyme inhibitor }\end{array}$ & $123(47)$ & $296(49)$ & 0.431 \\
\hline Aspirin & $216(82)$ & $477(80)$ & 0.484 \\
\hline
\end{tabular}

Data are given as absolute values with percent values in parentheses or mean $\pm \mathrm{SD}$

with fibrinogen were no longer significant. Virtually in all models, older age, female sex, higher BMI, positive history of $\mathrm{CHF}$, positive history of COPD, and current smoking were independent predictors of elevated CRP levels. Likewise, greater age, male sex, higher BMI, positive history of $\mathrm{CHF}$, and current smoking were independent predictors of elevated IL-6 levels in most models. Older age, higher BMI, history of diabetes, and current smoking were found to be independent predictors of elevated fibrinogen levels in most models.

Because age, BMI, and smoking status were independent predictors of CRP, IL-6, and fibrinogen, we additionally computed interaction terms for the various HRV measures with age, BMI, and smoking. However, we found no evidence that the relationship between decreased HRV and inflammation was moderated by age, BMI, or current smoking (all $p$ values for interaction $>0.10$ ).

\section{Discussion}

Cross-sectional studies in healthy populations have clearly demonstrated an independent association between HRV and both CRP and IL-6 levels [12-20]. However, until now, the association between HRV and inflammation in patients with CHD has been less clear. The largest prior study of patients with CHD found an association between time-domain measures of HRV and CRP in a sample of 269 patients with clinically suspected CAD who were referred for angiography [28]. Our study expands these findings by demonstrating a robust inverse association between time-domain measures of HRV and two inflammatory biomarkers (i.e., CRP and IL-6) in a large sample of 862 outpatients with stable CHD. Together with the results of prior studies, these findings make it clear that low HRV is indeed associated with inflammation in patients with stable CHD. We further found that HRV is inversely associated with fibrinogen, although less consistently so than with CRP and IL-6, when taking covariates into account. Nevertheless, our findings concur with studies in healthy populations [15, 21], depressed CHD patients [22], and women patients with CHD [27], all suggesting an inverse relationship between HRV and fibrinogen.

Research shows that the vagus nerve curtails inflammatory activity of peripheral tissue macrophages via an anticholinergic pathway [34]. The vagus nerve also informs the brain about peripheral inflammation resulting in activation of the hypothalamic pituitary adrenal axis with the subsequent increase in peripheral cortisol level having systemic anti-inflammatory effects [35]. HRV measures indicating vagal modulation of heart rate (e.g., RMSSD) thus might play a role in systemic inflammation related to atherosclerosis and CHD [36]. However, we found no evidence for a significant relationship between RMSSD, an index of respiratory sinus arrhythmia, and inflammation. One explanation might be that some measures of vagal cardiac control tend to decline with age and thus, even when controlling for age statistically, RMSSD might not reliably reflect vagal modulation of heart rate in our group 
Table 2 Association of heart rate variability measures with inflammatory markers

\begin{tabular}{|c|c|c|c|c|c|c|c|c|c|c|c|c|}
\hline \multirow[t]{3}{*}{ HRV measure } & \multicolumn{4}{|c|}{ Log interleukin-6 } & \multicolumn{4}{|c|}{ Log fibrinogen } & \multicolumn{4}{|c|}{ Log C-reactive protein } \\
\hline & \multicolumn{2}{|c|}{ Model 1} & \multicolumn{2}{|c|}{ Model 2} & \multicolumn{2}{|c|}{ Model 1} & \multicolumn{2}{|c|}{ Model 2} & \multicolumn{2}{|c|}{ Model 1} & \multicolumn{2}{|c|}{ Model 2} \\
\hline & $r$ & $p$ value & $r$ & $p$ value & $r$ & $p$ value & $r$ & $p$ value & $r$ & $p$ value & $r$ & $p$ value \\
\hline Mean NN (ms) & -0.11 & 0.002 & -0.08 & 0.017 & -0.08 & 0.026 & -0.03 & 0.415 & -0.16 & $<0.001$ & -0.11 & 0.003 \\
\hline SDNN (ms) & -0.19 & $<0.001$ & -0.14 & $<0.001$ & -0.12 & 0.001 & -0.06 & 0.105 & -0.14 & $<0.001$ & -0.08 & 0.016 \\
\hline SDANN (ms) & -0.18 & $<0.001$ & -0.14 & $<0.001$ & -0.11 & 0.001 & -0.05 & 0.152 & -0.14 & $<0.001$ & -0.08 & 0.020 \\
\hline RMSSD (ms) & -0.02 & 0.568 & -0.02 & 0.577 & -0.03 & 0.431 & -0.01 & 0.788 & $<0.01$ & 0.965 & $<0.01$ & 0.918 \\
\hline
\end{tabular}

Model 1: adjusted for age

Model 2: adjusted for age, sex, race (White vs. non-White), body mass index, use of beta blockers, history of diabetes, history of congestive heart failure, history of chronic obstructive pulmonary disease, and current smoking

Shown are partial correlation coefficients with $p$ values

Fig. 1 Mean \pm SE ageadjusted levels of inflammatory markers in patients with low (SDNN < 100) versus normal $($ SDNN $\geq 100)$ heart rate variability. The bar graphs (ac) illustrate the age-adjusted mean \pm SEM levels of interleukin-6, fibrinogen, and Creactive protein levels in patients with SDNN $<100 \mathrm{~ms}$ (low heart rate variability, HRV) compared to those with $\mathrm{SDNN} \geq 100 \mathrm{~ms}$ (normal HRV). Data are given in original units (all $p<0.05$ )

\section{(A) Interleukin-6}

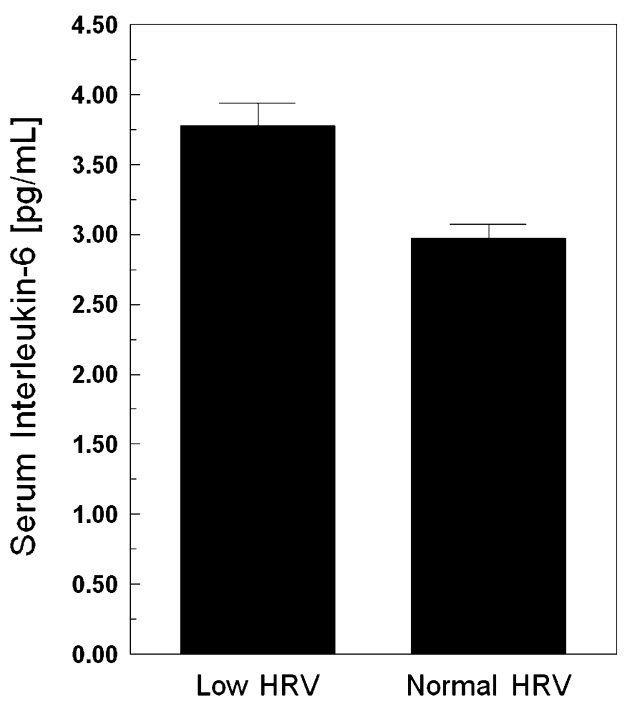

(B) Fibrinogen

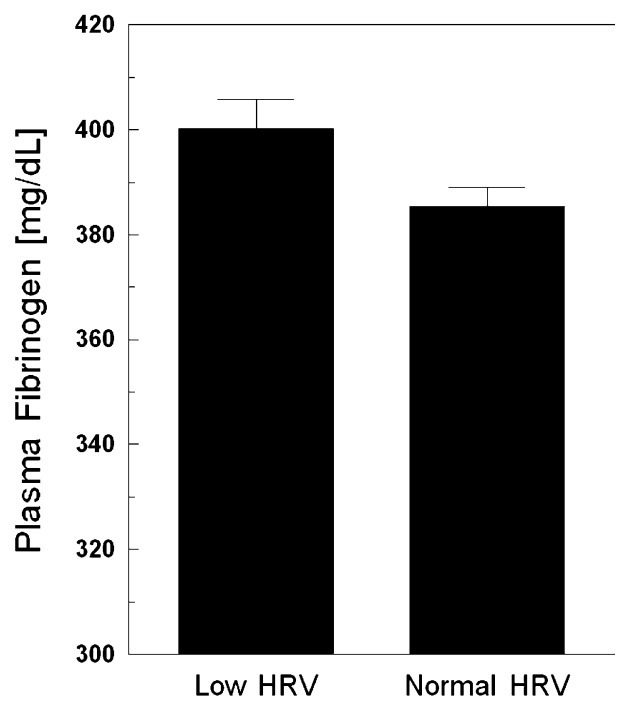

(C) C-reactive protein

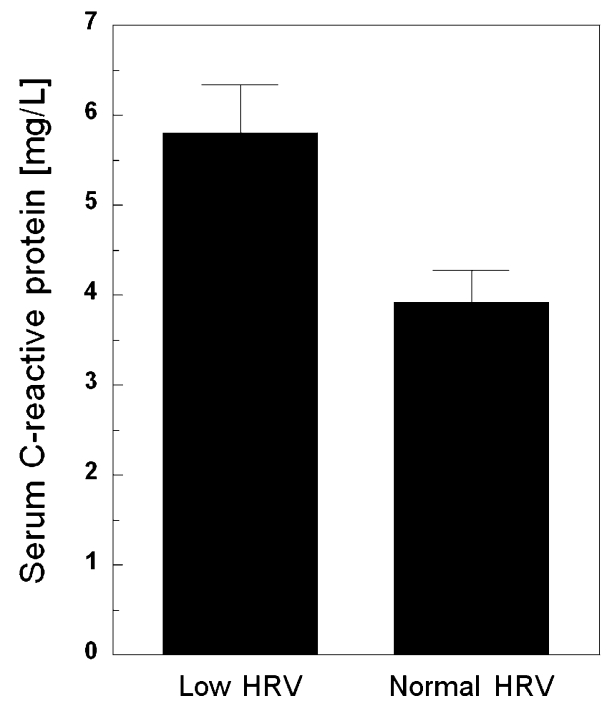


of relatively older individuals. To support this notion, our group found in middle-aged healthy subjects an inverse relationship between night-time RMSSD and levels of both CRP [17] and fibrinogen [21] and between $\mathrm{HF}$ power (another index of vagal cardiac control) and IL-6 [20] after controlling for covariates. It is also likely that CHD itself affects RMSSD through erratic rhythms as shown in older subjects with cardiovascular disease [37].

The cross-sectional nature of our study precludes any inference about the causal direction of the relationship between HRV and inflammation. Therefore, our findings may suggest that inflammation contributes to the poor prognosis associated with low HRV in patients with CHD on one hand. On the other hand, the findings may also suggest that low HRV contributes to poor CHD prognosis associated with inflammatory markers. In subjects with no apparent heart disease, the combination of decreased HRV and increased CRP has been shown to synergistically predict future risk of first-time MI and all-cause mortality [38]. Decreased HRV also predicted cardiovascular death during 13-year follow-up in patients with mild-moderate heart failure [39], and inflammation (incl. CRP) has been shown to be associated with markers of heart failure severity [40]. However, it is as yet unclear whether decreased HRV leads to inflammation or vice versa. Nevertheless, the sustained increase in IL-6 with decreased HRV might further plaque progression and instability, thereby increasing the risk of plaque rupture and coronary thrombosis [41, 42]. The HRV-associated inflammation also seems to be involved in the acute phase of a CHD event. This is evidenced by inverse associations between HRV and both IL-6 and CRP in patients with acute coronary syndromes [43, 44], whereby patients with complicated acute coronary syndromes show particularly high levels of proinflammatory cytokines, including IL-6 [45]. One possible limitation of our study is that we pooled data from diabetics and non-diabetics. Although we adjusted our final model for diabetes, diabetics with their neuropathy and non-diabetics have different conditions affecting HRV, which might also affect results.

In conclusion, we have substantiated the current assumption of an inverse relationship between HRV and chronic low-grade systemic inflammation in patients with stable CHD. The independent relationship between compromised cardiac autonomic control and increased circulating levels of CRP, IL-6, and to a lesser extent fibrinogen, might play an important role in the progression of $\mathrm{CHD}$ and recurrent events.

Acknowledgments The Heart and Soul Study was supported by grants from the Department of Veterans Affairs (Epidemiology Merit Review Program), Washington, DC; The Robert Wood Johnson Foundation (Generalist Physician Faculty Scholars Program), Princeton, New Jersey; the American Federation for Aging Research
(Paul Beeson Faculty Scholars in Aging Research Program), New York, NY; and the Ischemia Research and Education Foundation, San Bruno, CA.

\section{References}

1. Libby P, Theroux P (2005) Pathophysiology of coronary artery disease. Circulation 111:3481-3488

2. Danesh J, Wheeler JG, Hirschfield GM et al (2004) C-reactive protein and other circulating markers of inflammation in the prediction of coronary heart disease. $\mathrm{N}$ Engl J Med 350:1387-1397

3. Danesh J, Kaptoge S, Mann AG et al (2008) Long term interleukin-6 levels and subsequent risk of coronary heart disease: two new prospective studies and a systematic review. PLoS Med $5: \mathrm{e} 78$

4. Fibrinogen Studies Collaboration (2005) Plasma fibrinogen level and the risk of major cardiovascular diseases and nonvascular mortality: an individual participant meta-analysis. JAMA 294:1799-1809

5. Khuseyinova N, Koenig W (2006) Biomarkers of outcome from cardiovascular disease. Curr Opin Crit Care 12:412-419

6. Bogaty P, Poirier P, Simard S, Boyer L, Solymoss S, Dagenais GR (2001) Biological profiles in subjects with recurrent acute coronary events compared with subjects with long standing stable angina. Circulation 103:3062-3068

7. Ridker PM, Rifai N, Pfeffer MA et al (1998) Inflammation, pravastatin, and the risk of coronary events after myocardial infarction in patients with average cholesterol levels. Cholesterol and Recurrent Events (CARE) Investigators. Circulation 98:839-844

8. Haim M, Benderly M, Tanne D et al (2007) C-reactive protein, bezafibrate, and recurrent coronary events in patients with chronic coronary heart disease. Am Heart J 154:1095-1101

9. Sinning JM, Bickel C, Messow CM et al (2006) Impact of Creactive protein and fibrinogen on cardiovascular prognosis in patients with stable angina pectoris: the AtheroGene study. Eur Heart J 27:2962-2968

10. Biasucci LM, Vitelli A, Liuzzo G et al (1996) Elevated levels of interleukin-6 in unstable angina. Circulation 94:874-877

11. Haensel A, Mills PJ, Nelesen RA, Ziegler MG, Dimsdale JE (2008) The relationship between heart rate variability and inflammatory markers in cardiovascular diseases. Psychoneuroendocrinology 33:1305-1312

12. Kon H, Nagano M, Tanaka F, Satoh K, Segawa T, Nakamura M (2006) Association of decreased variation of R-R interval and elevated serum C-reactive protein level in a general population in Japan. Int Heart J 47:867-876

13. Araújo F, Antelmi I, Pereira AC et al (2006) Lower heart rate variability is associated with higher serum high-sensitivity Creactive protein concentration in healthy individuals aged 46 years or more. Int J Cardiol 107:333-337

14. Felber Dietrich D, Schindler C, Schwartz J et al (2006) Heart rate variability in an ageing population and its association with lifestyle and cardiovascular risk factors: results of the SAPALDIA study. Europace 8:521-529

15. Chuang KJ, Chan CC, Su TC, Lee CT, Tang CS (2007) The effect of urban air pollution on inflammation, oxidative stress, coagulation, and autonomic dysfunction in young adults. Am J Respir Crit Care Med 176:370-376

16. Lampert R, Bremner JD, Su S et al (2008) Decreased heart rate variability is associated with higher levels of inflammation in middle-aged men. Am Heart J 156:759.e1-e7

17. Thayer JF, Fischer JE (2009) Heart rate variability, overnight urinary norepinephrine and $\mathrm{C}$-reactive protein: evidence for the 
cholinergic anti-inflammatory pathway in healthy human adults. J Intern Med 265:439-447

18. Sloan RP, McCreath H, Tracey KJ, Sidney S, Liu K, Seeman T (2007) RR interval variability is inversely related to inflammatory markers: the CARDIA study. Mol Med 13:178-184

19. Stein PK, Barzilay JI, Chaves PH et al (2008) Higher levels of inflammation factors and greater insulin resistance are independently associated with higher heart rate and lower heart rate variability in normoglycemic older individuals: the Cardiovascular Health Study. J Am Geriatr Soc 56:315-321

20. von Känel R, Nelesen RA, Mills PJ, Ziegler MG, Dimsdale JE (2008) Relationship between heart rate variability, interleukin-6, and soluble tissue factor in healthy subjects. Brain Behav Immun 22:461-468

21. von Känel R, Thayer JF, Fischer JE (2009) Nighttime vagal cardiac control and plasma fibrinogen levels in a population of working men and women. Ann Noninvasive Electrocardiol 14:176-184

22. Carney RM, Freedland KE, Stein PK et al (2007) Heart rate variability and markers of inflammation and coagulation in depressed patients with coronary heart disease. J Psychosom Res 62:463-467

23. Nolan RP, Reid GJ, Seidelin PH, Lau HK (2007) C-reactive protein modulates vagal heart rate control in patients with coronary artery disease. Clin Sci 112:449-456

24. Santangeli P, Sgueglia GA, Sestito A et al (2008) Different effect of percutaneous and surgical coronary revascularization on cardiac autonomic function and inflammation in patients with stable angina. Int J Cardiol 127:269-270

25. Yue W, Schneider A, Rückerl R et al (2007) Relationship between electrocardiographic and biochemical variables in coronary artery disease. Int J Cardiol 119:185-191

26. Janszky I, Ericson M, Lekander M et al (2004) Inflammatory markers and heart rate variability in women with coronary heart disease. J Intern Med 256:421-428

27. von Känel R, Orth-Gomér K (2008) Autonomic function and prothrombotic activity in women after an acute coronary event. J Womens Health 17:1331-1337

28. Madsen T, Christensen JH, Toft E, Schmidt EB (2007) C-reactive protein is associated with heart rate variability. Ann Noninvasive Electrocardiol 12:216-222

29. Ruo B, Rumsfeld JS, Hlatky MA, Liu H, Browner WS, Whooley MA (2003) Depressive symptoms and health-related quality of life: the Heart and Soul Study. JAMA 290:215-221

30. Bush K, Kivlahan DR, McDonell MB, Fihn SD, Bradley KA (1998) The AUDIT alcohol consumption questions (AUDIT-C): an effective brief screening test for problem drinking Ambulatory Care Quality Improvement Project (ACQUIP). Alcohol Use Disorders Identification Test. Arch Intern Med 158:1789-1795

31. (1996) Heart rate variability: standards of measurement, physiological interpretation and clinical use. Task Force of the European
Society of Cardiology and the North American Society of Pacing and Electrophysiology. Circulation 93:1043-1065

32. Kleiger RE, Miller JP, Bigger JT Jr, Moss AJ (1987) Decreased heart rate variability and its association with increased mortality after acute myocardial infarction. Am J Cardiol 59:256-262

33. Gehi A, Mangano D, Pipkin S, Browner WS, Whooley MA (2005) Depression and heart rate variability in patients with stable coronary heart disease: findings from the Heart and Soul Study. Arch Gen Psychiatry 62:661-666

34. Tracey KJ (2002) The inflammatory reflex. Nature 420:853-859

35. Gidron Y, Kupper N, Kwaijtaal M, Winter J, Denollet J (2007) Vagus-brain communication in atherosclerosis-related inflammation: a neuroimmunomodulation perspective of CAD. Atherosclerosis 195:e1-e9

36. Thayer JF (2009) Vagal tone and the inflammatory reflex. Cleve Clin J Med 76(Suppl 2):S23-S26

37. Stein PK, Domitrovich PP, Hui N, Rautaharju P, Gottdiener J (2005) Sometimes higher heart rate variability is not better heart rate variability: results of graphical and nonlinear analyses. J Cardiovasc Electrophysiol 16:954-959

38. Sajadieh A, Nielsen OW, Rasmussen V, Hein HO, Hansen JF (2006) C-reactive protein, heart rate variability and prognosis in community subjects with no apparent heart disease. J Intern Med 260:377-387

39. Smilde TD, van Veldhuisen DJ, van den Berg MP (2009) Prognostic value of heart rate variability and ventricular arrhythmias during 13-year follow-up in patients with mild to moderate heart failure. Clin Res Cardiol 98:233-239

40. Jensen J, Ma LP, Fu ML, Svaninger D, Lundberg PA, Hammarsten O (2010) Inflammation increases NT-proBNP and the NT-proBNP/BNP ratio. Clin Res Cardiol 99:445-452

41. Lobbes MB, Lutgens E, Heeneman S et al (2006) Is there more than $\mathrm{C}$-reactive protein and fibrinogen? The prognostic value of soluble CD40 ligand, interleukin-6 and oxidized low-density lipoprotein with respect to coronary and cerebral vascular disease. Atherosclerosis 187:18-25

42. Ridker PM, Rifai N, Stampfer MJ, Hennekens CH (2000) Plasma concentration of interleukin- 6 and the risk of future myocardial infarction among apparently healthy men. Circulation 101:1767-1772

43. Hamaad A, Sosin M, Blann AD, Patel J, Lip GY, MacFadyen RJ (2005) Markers of inflammation in acute coronary syndromes: association with heart rate and reductions in heart rate variability. Clin Cardiol 28:570-576

44. Lanza GA, Sgueglia GA, Cianflone D et al (2006) Relation of heart rate variability to serum levels of C-reactive protein in patients with unstable angina pectoris. Am $\mathrm{J}$ Cardiol 97:1702-1706

45. Debrunner M, Schuiki E, Minder E et al (2008) Proinflammatory cytokines in acute myocardial infarction with and without cardiogenic shock. Clin Res Cardiol 97:298-305 